\title{
Flat Tympanometry Result
}

National Cancer Institute

\section{Source}

National Cancer Institute. Flat Tympanometry Result. NCI Thesaurus. Code C88507.

A tympanometry test result indicating the lack of movement of the tympanic membrane due to middle ear pathology. 